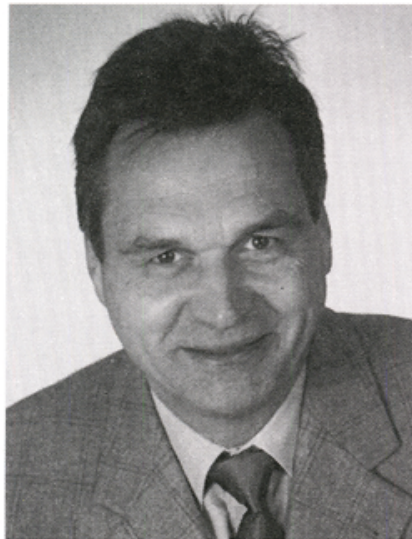

Uwe Fichtner

\section{Jahr der Geowissenschaften}

In diesem Jahr finden in Deutschland zahlreiche Veranstaltungen unter einem gemeinsamen Motto und mit einem eigens dafür kreierten logo "planet erde" statt. Wissenschaftlerinnen und Wissenschaftler präsentieren Forschung publikumsnah zum "Anfassen". Die vier Begriffe "Fever, Wasser, Erde, Luft" stehen symbolisch für Forschungsgegenstand und Arbeitsfelder der Geowissenschaften, das "System Erde" mit seinen Komponenten Geosphäre, Hydro- und Kryosphäre, Biosphäre und Atmosphäre. Das Themenspektrum reicht von der Entstehung der Erde über Stoffkreisläufe, Naturgefahren und Rohstoffsicherung bis zur Debatte um klimatische Veränderungen. Um den Dialog zwischen Wissenschaft und O'ffentlichkeit zu fördern, hat die Bundesministerin für Bildung und Forschung, Edelgard Bulmahn, gemeinsam mit dem Stifterverband und den großen Forschungsorganisationen 2002 zum Jahr der Geowissenschaften ausgerufen Is. Peter Aschenberner, Präsidenten der DGfK auf S. 811.

"Machen Sie mit und besuchen Sie eine Veranstaltung in Ihrer Region oder nehmen Sie mit Ihrem Institut, Ihrer Organisation oder Ihrem Verein an einer Veranstaltung teil!" - so der Aufruf der Initiatoren. Inzwischen findet man 254 Veranstaltungen unter der Internet-Adresse http://www. planeterde.de/Veranstaltungen/ Regional/Kalender/Uebersicht aufgelistet, und laufend werden es mehr. Wissenschaftler, Institute, Sektionen und Kommissionen der DGFK, die sich noch beteiligen möchten oder auf einer Science Street ihre Forschungsergebnisse der Offfentlichkeit vorstellen wollen, können sich bei der zentralen Koordinierungsstelle am Geo-Forschungs-Zentrum Potsdam (GFZ) unter der o.g. Homepage eintragen und anmelden. Unser Fach ist mit eigenen Veranstaltungen vertreten (s. Veranstaltungskalender in diesem Heft auf S. 95I und gerade in diesen Tagen findet eine Kartenausstellung zum Weltbild der frühen Neuzeit in der Universitätsbibliothek in Göttingen statt.

Die Zielsetzung einer verbesserten Kommunikation zwischen Wissen Schaffenden und Wissen Nutzenden geht über bildungspolitische Aspekte hinaus; sie berührt auf inhaltlicher Ebene zugleich auch die Kartographie, die mit ihren Fähigkeiten zur Visualisierung von Daten und Erkenntnissen gerade für die Geowissenschaften als methodisches Instrument von großem Belang ist. Allenthalben werden in den Vorträgen und Veranstaltungen analoge und digitale Karten oder kartenverwandte Darstellungen zu sehen sein und die Ergebnisse von GIS-Analysen präsentiert werden. Um so wichtiger ist es, das die Kartographie in der Kommunikation mit anderen Fachgebieten oder der Offentlichkeit die richtigen Signale setzt, in denen Kompetenz und Inhalte angemessen zum Ausdruck kommen. In diesem Zusammenhang spielen auch Begriffe und Bezeichnungen eine Rolle, weshalb die Schriffleitung in diesem Heft an Sie, liebe Leserinnen und Leser, die Frage richtet, ob der Name unserer Fachzeitschrift noch zeitgerecht ist oder geändert werden müsste (s. Beitrag auf S. 77).

Um den Kommunikationsprozess zwischen Karte und Kartennutzer geht es im Beitrag von Lars Brodersen u.a. Sicherlich liegt der Studie nur eine sehr kleine Stichprobe zugrunde, so dass manche Schlüsse vielleicht als zu unsicher erscheinen mögen. Interessant ist ihr Ansatz allemal, übernimmt er doch eine Methode, die aus der Markfforschung mit Untersuchungen zur Perzeption von Anzeigen bekannt geworden ist. Zum Jahr der Geowissenschaften passt der Aufsatz von Gotthard Meinel und Anja Lange.

Ihr

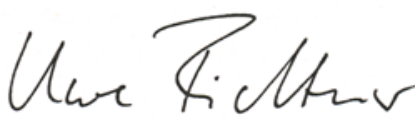

